\title{
Oral vibrotactile screening: Reliability of low-frequency lingual vibrotactile thresholds obtained for two baseline conditions
}

\author{
KAL M. TELAGE and EMILY POWELL \\ Ithaca College, Ithaca, New York \\ PATTI DENMEADE \\ Neurologic Center at Cortland, Cortland, New York \\ and \\ MARGIE COURTNEY \\ Ithaca College, Ithaca, New York
}

\begin{abstract}
The investigation evaluated the reliability of lingual vibrotactile thresholds obtained across two baseline conditions separated by an average of 14 days. Each baseline condition included three awareness training procedures as well as determination of the median of three vibrotactile thresholds. Results indicated a high degree of individual and intergroup threshold consistency. The authors view the technique as a possible screening procedure for general diagnostic use with adults. A discussion featuring specific future research needs is included.
\end{abstract}

The normal sensitivity of lingual tissues to a vibrotactile stimulus has been of interest to speech scientists for more than a decade. This interest derives from the fact that many theories about speech motor control emphasize the importance of haptic sensory feedback mechanisms (Fairbanks, 1954; Mysak, 1966; Van Riper \& Irwin, 1962). Early research in this area attempted to determine normative levels for lingual vibrotactile thresholds (Fucci, 1972; Fucci, Hall, \& Weiner, 1971; Telage \& Fucci, 1974; Telage, Fucci, \& Arnst, 1972). In general, these studies provided data about lingual sensitivity based on single-trial measurements; the exception was Telage and Fucci's (1974) study, which provided normative data for successive measurements. Furthermore, these investigations measured vibrotactile thresholds within a range of higher frequencies than was used in the present investigation.

Recent findings by Telage and Gorman (1985) indicated considerably greater lingual vibrotactile threshold detectability in the $30-50-\mathrm{Hz}$ frequency range. Because the earlier studies cited above were concerned primarily with obtaining thresholds at frequencies at or greater than $200 \mathrm{~Hz}$, normative data relative to lower, more detectable frequencies is presently unavailable.

The purpose of the present study was to obtain normative baselines for low-frequency lingual vibrotactile thresholds that reflect both individual and group response patterns.

Address correspondence to Kal M. Telage, Department of SpeechLanguage Pathology and Audiology, School of Allied Health, Ithaca College, Ithaca, NY 14850.

\section{METHOD}

\section{Subjects}

Nine young adults with no history of speech or sensory-motor impairments served as subjects for this investigation. Initially, all subjects were familiarized with the instrumentation and received an explanation of the purpose of the study and the nature of the stimulus presented.

\section{Apparatus}

Figure 1 presents a block diagram of the instrument package used in this study. The stimulus control unit is composed of Coulbourn solidstate logic modules. These units generate pulsed vibratory signals that may be varied in frequency, intensity, and temporal characteristics. Three universal timers are programmed to control signal duration and duty cycle. The timers gate a selectable rise-fall module on and off. This continuously adjustable electronic switch is set to generate a rise-fall time of $100 \mathrm{msec}$. The signal from the rise-fall module is fed into a precision signal generator and an audio mixer amplifier. Stimulus intensities are varied in increments of $256.5-\mathrm{dB}$ steps by passing them through a programmable attenuator and an 8-bit binary up-down counter. Pulsed signals from the stimulus control unit drive the electromagnetic minivibrator that is the stimulus-producing aggregate of the system.

\section{Testing Procedure}

Figure 2 presents a schematic diagram of the vibrator and free-surround disk. During threshold measurements, each subject reclined in a motorized dental lounge adjusted for easy access to the vibrator apparatus. With the head reclining and supported, the lounge was elevated to allow the subject to press the anterior midline surface of the tongue against the surround disk. While the subject was in this position, the contactor $\left(.32 \mathrm{~cm}^{2}\right)$ was lowered through the opening in the disk until it extended $1 \mathrm{~mm}$ into the lingual surface. The diameter of the opening in the upper surround disk was $2 \mathrm{~mm}$ larger than the diameter of the contactor.

\section{Awareness Training}

Awareness training procedures were employed for all subjects prior to baseline threshold determinations for Trials 1 and 2 . Awareness training included three steps during which subjects tracked the lingual vibra- 


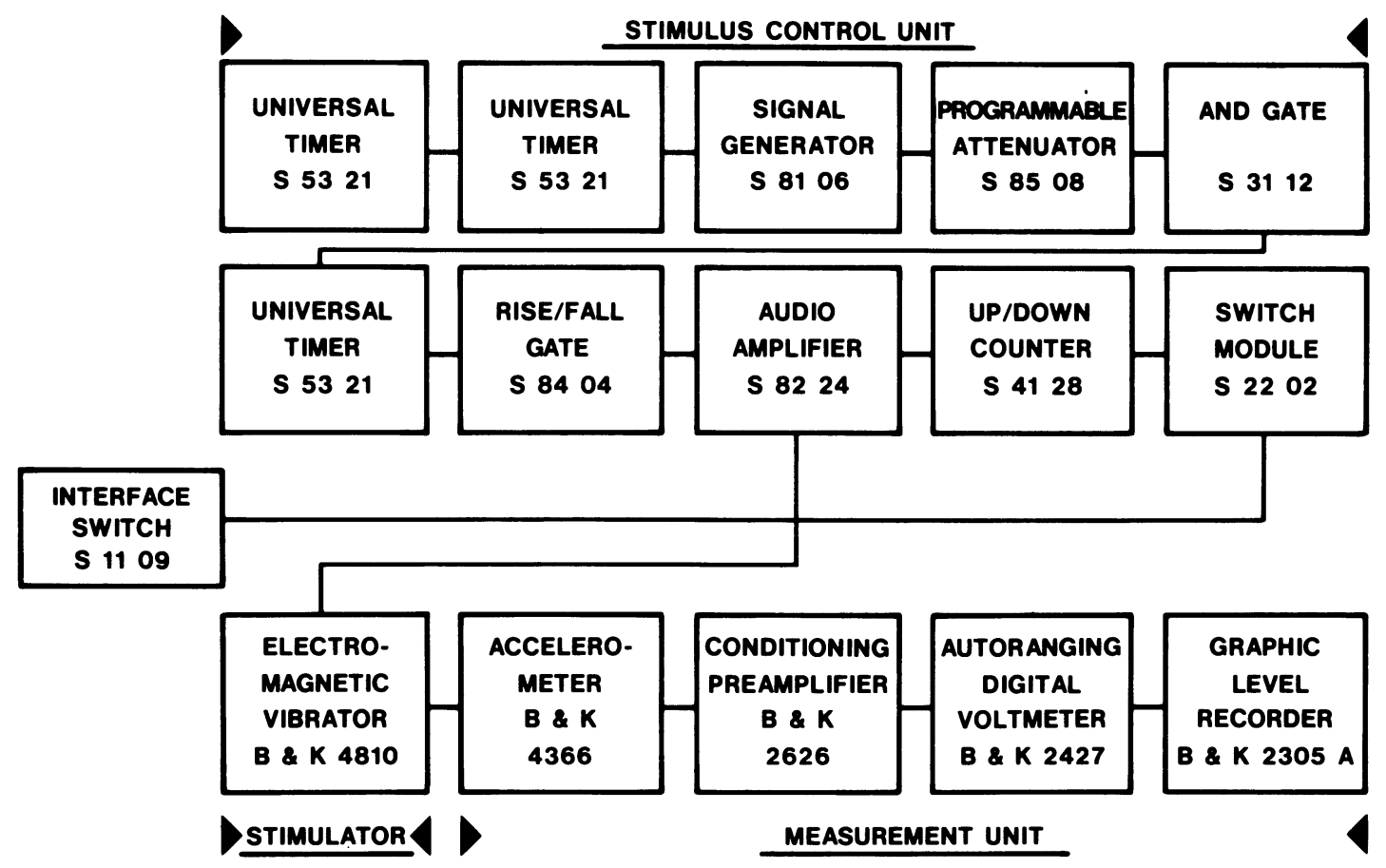

Figure 1. Block diagram of the instrumentation package.

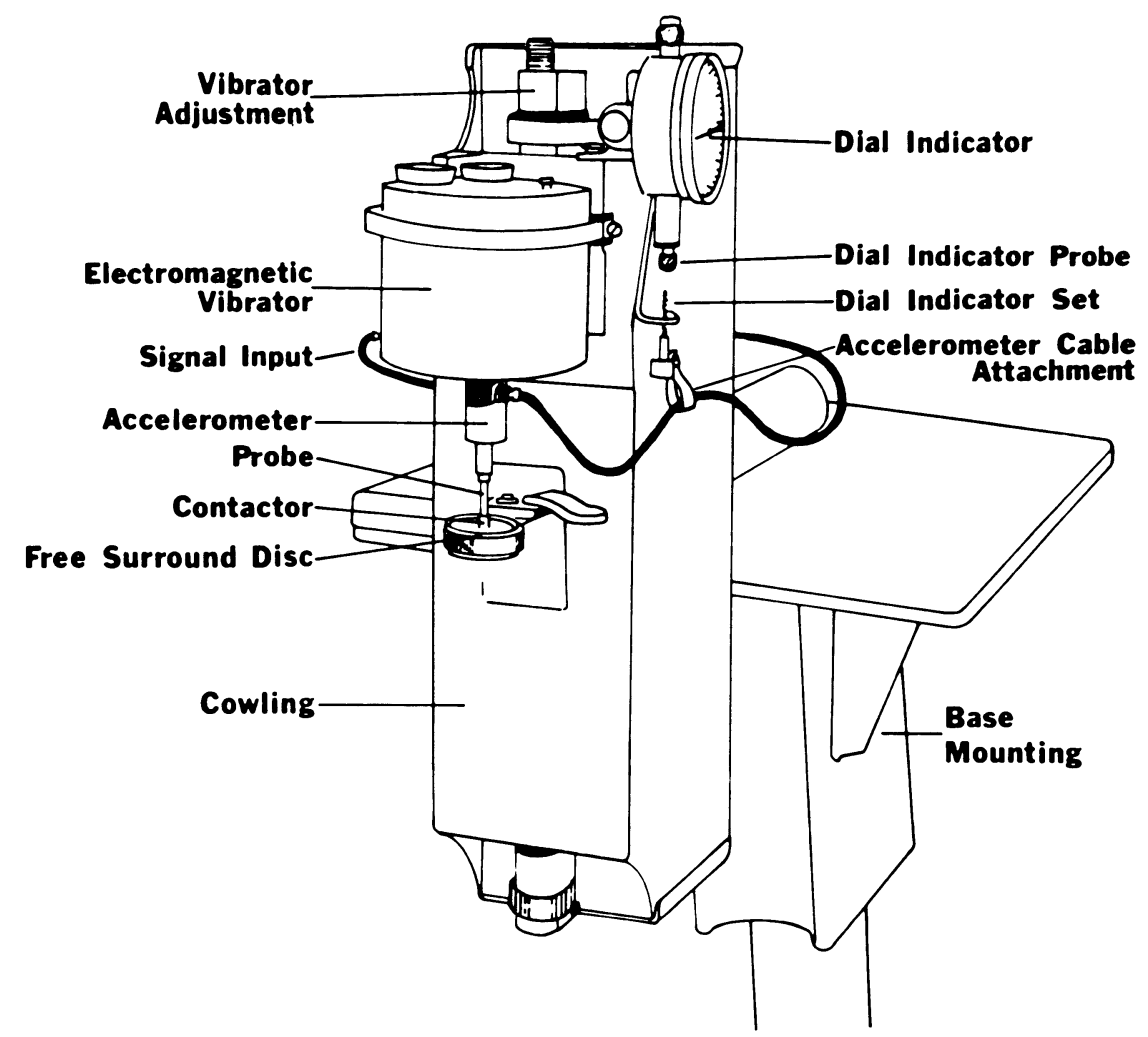

Figure 2. Schematic diagram of the free-surround disk and vibratory portions of the oral vibrotactile stimulator. 
tory stimulus at suprathreshold levels. Step 1 required subjects to focus their complete attention on a $50-\mathrm{Hz}$ pulsed vibratory stimulus that began at $100 \mathrm{mV}$ of contactor displacement and was automatically attenuated in 5-mV steps, using a descending psychophysical method of limits. During this procedure, the intensity of the vibrotactile stimulus remained well above each subject's threshold. Subjects closed their eyes and attended to the tingling vibratory sensation, which was localized at the anterior midline area of the tongue by the upper surround disk.

During Step 2, vibrotactile awareness training began at an intensity of $50 \mathrm{mV}$ and decreased in steps of $2 \mathrm{mV}$ per pulse to a level of $18 \mathrm{mV}$. During Step 3, the second awareness trial began at an intensity level of $30 \mathrm{mV}$ and was attenuated in the same manner to a level of $14 \mathrm{mV}$. Both awareness procedures were presented at suprathreshold levels. The purpose was to familiarize subjects with the test procedures and orient them to tracking the vibratory stimulus.

\section{Baseline Conditions}

Immediately after the two awareness trials, three successive lingual vibrotactile thresholds were obtained from each subject as Baseline Condition 1. Pulsed stimuli (on $1.0 \mathrm{sec}$, off $1.0 \mathrm{sec}$ ) were initially presented at $15 \mathrm{mV}$ of contactor displacement and were automatically attenuated in steps of approximately $2 \mathrm{mV}$ per pulse, using a descending psychophysical method of limits. Subjects pressed an interface switch when they no longer detected the vibrotactile stimulus. The complete procedure, including awareness training and threshold determination, was repeated for each subject as Baseline Condition 2. Conditions 1 and 2 were separated by an average latency of 2 weeks.

\section{Threshold Measurement}

An accelerometer mounted on the vibrating probe was set to emit $49.5 \mathrm{mV} / \mathrm{g}$ of acceleration as a function of contactor displacement. Displacement voltages from the accelerometer were amplified and then monitored by a digital voltmeter set to read peak displacement values in millivolts. The median of three threshold trials was accepted as each subject's baseline threshold for Conditions 1 and 2 .

\section{RESULTS AND DISCUSSION}

Table 1 presents means and standard deviations for lingual vibrotactile baseline thresholds obtained for each condition. The threshold means for all subjects are very consistent and differ by only $0.4 \mathrm{mV}$ between conditions. A $t$ test for related means indicated a nonsignificant difference at the .01 level of confidence. Standard deviations of 1.0 and $1.5 \mathrm{mV}$ reflect highly consistent threshold responses. These findings are also consistent with lowfrequency vibrotactile threshold data reported by Telage and Gorman (1985).

Figure 3 compares each subject's median vibrotactile threshold for Trials 1 and 2 to determine the extent of individual threshold variability between assessments. Differences between successive thresholds range from 0.3 to $1.8 \mathrm{mV}$. Six of the 9 subjects' two thresholds differed by less than $1.0 \mathrm{mV} ; 2$ subjects' successive thresholds

Table 1

Means and Standard Deviations for Lingual Vibrotactile Thresholds Expressed in Millivolts of Peak Displacement $(N=9)$

\begin{tabular}{ccc}
\hline & $\begin{array}{c}\text { Baseline Condition 1 } \\
50 \mathrm{~Hz}\end{array}$ & $\begin{array}{c}\text { Baseline Condition 2 } \\
50 \mathrm{~Hz}\end{array}$ \\
\hline$M$ & 3.7 & 4.1 \\
$S D$ & 1.0 & 1.5 \\
$t$ & $4.93^{*}$ & \\
\hline
\end{tabular}

*Nonsignificant at the .01 level of confidence.

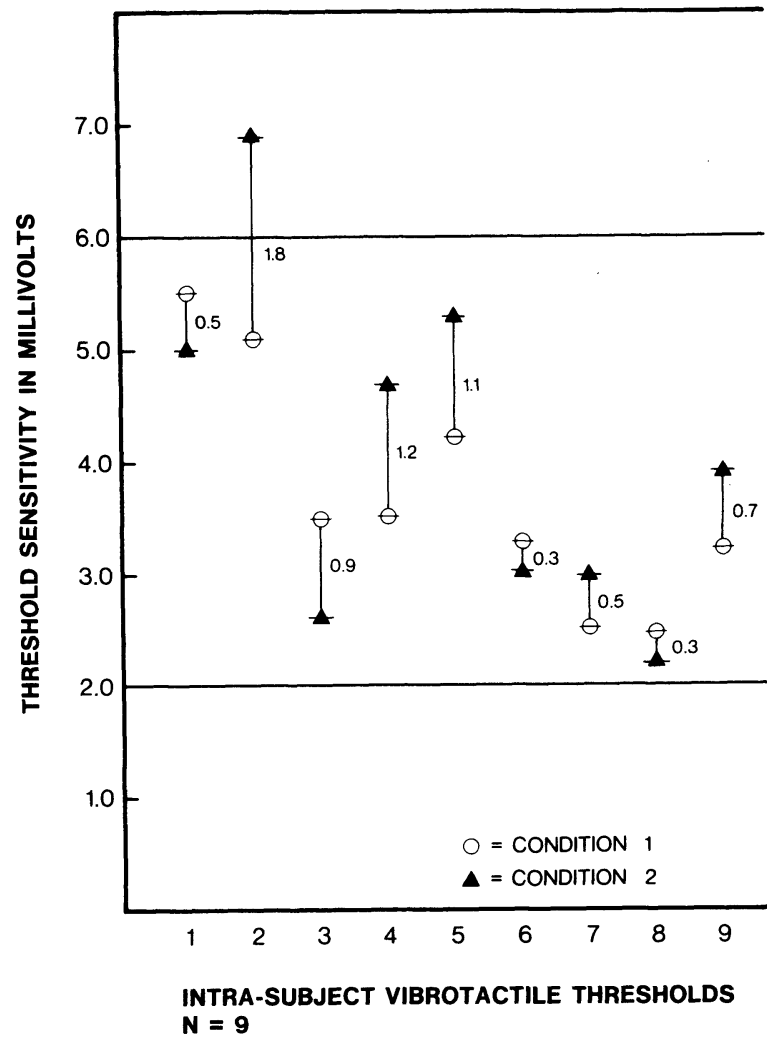

Figure 3. Intrasubject vibrotactile threshold data for Conditions 1 and $2(N=9)$.

differed by 1.1 and $1.2 \mathrm{mV}$. The thresholds of only 1 subject varied by as much as $1.8 \mathrm{mV}$ between the two trials.

In determining the consistency of baseline thresholds, it is interesting to convert the range of threshold responses in millivolts to a measure that indicates actual linear displacements of the vibrating contactor into the lingual tissues. Trial 1 thresholds ranged from a low of 2.5 to a high of $5.5 \mathrm{mV}$. Trial 2 thresholds ranged from a low of 2.2 to a high of $6.9 \mathrm{mV}$. The overall range in threshold sensitivity across both trials was 2.2 to $6.9 \mathrm{mV}$. The following formula is used to convert the overall threshold range (2.2 to $6.9 \mathrm{mV})$ to microns of peak contactor displacement:

$$
\begin{aligned}
\text { Microns } & =\mu \mathrm{V} \times 250,000 \\
& =F^{2} \times(\text { sensitivity of accelerometer in } \mu \mathrm{V} / \mathrm{g}=49.5) .
\end{aligned}
$$

This conversion produces thresholds that range from 4.4 to $13.8 \mu$ of peak displacement. Since $1 \mu=$ one thousandth of a millimeter, it is clear that subjects' baseline thresholds occurred within a range of 4.4 to 13.8 thousandths of a millimeter of peak contactor displacements into the lingual surface. This appears to be a very narrow range of differential displacements and suggests a highly sensitive and consistent neural mechanoreceptive response. 
From a clinical standpoint, the method used-including initial subject orientation to the nature and locus of the vibrotactile stimulus, the two suprathreshold awareness training trials, and determination of the median of three successive thresholds-appears to serve as a reliable lingual vibrotactile screening procedure for use with adult subjects. On the basis of the present findings, normal adult subjects should have lingual vibrotactile thresholds at $50 \mathrm{~Hz}$ that range between 2.0 and $7.0 \mathrm{mV}$, with the average falling somewhere around $4.0 \mathrm{mV}$.

Continued research is indicated to support the tentative conclusions based on this investigation. It is necessary to obtain a larger number of successive baseline threshold trials per subject and to extend them over a longer time interval. Continued research might also be directed toward determining whether this screening procedure can be used to obtain reliable thresholds from children. This could lead to comparisons of children's and adults' thresholds that may identify changes in lingual sensitivity that occur with early maturation and/or aging.

Finally, if this screening procedure or modifications of it continue to be reliable measures of lingual vibrotactile sensitivity, research efforts should be directed toward assessments within speech-disordered groups, particularly those with known or suspected neurogenic etiologies. The ability to identify the nature and extent of tactile sensory deficits that contribute to motor speech impairments has wide-ranging theoretical and clinical implications.

\section{REFERENCES}

FaIRBanKs, G. (1954). Systematic research in experimental phonetics: 1. A theory of the speech mechanism as a servosystem. Journal of Speech \& Hearing Disorders, 19, 133-139.

FuccI, D. J. (1972). Oral vibrotactile sensation: An evaluation of normal and defective speakers. Journal of Speech \& Hearing Research, 15, 179-184.

Fucci, D. J., Hall, D. E., \& WeINER, F. F. (1971). Normative study of oral and non-oral structures using vibrotactile stimuli. Perceptual Motor Skills, 33, 1099-1105.

MYSAK, E. D. (1966). Speech pathology and feedback theory. Springfield, IL: Charles C. Thomas.

TelaGe, K. M., \& FUCCI, D. J. (1974). Concerning intrasubject measurements of successive lingual vibrotactile responses. Perceptual Motor Skills, 39, 1047-1052.

Telage, K. M., FucCI, D. J., \& ARNST, D. J. (1972). Normative study of oral vibrotactile sensitivity. Perceptual Motor Skills, 35, 671-676.

Telage, K. M., \& Gorman, P. J. (1985). An investigation of lingual vibrotactile detectability. Bulletin of the Psychonomic Society, 23, 50-52.

VAN RIPER, C., \& IRWIN, J. (1962). Voice and articulation. Englewood Cliffs, NJ: Prentice-Hall.

(Manuscript received for publication March 9, 1987.) 